\title{
Risk factors for esophageal cancer: emphasis on infectious agents
}

\author{
Hala El-Zimaity, ${ }^{1}$ Vincenzo Di Pilato, ${ }^{2}$ Maria Novella Ringressi, ${ }^{3,4}$ Iva Brcic, ${ }^{5}$ \\ Shanmugarajah Rajendra, ${ }^{6,7,8}$ Rupert Langer, ${ }^{9}$ Bastian Dislich, ${ }^{10}$ Monika Tripathi, ${ }^{11}$ \\ Maha Guindi, ${ }^{12}$ and Robert Riddell ${ }^{13}$ \\ ${ }^{1}$ Dynacare Laboratories, Brampton, Ontario, Canada. ${ }^{2}$ Department of Clinical and Experimental Medicine, University of \\ Florence, Florence, Italy. ${ }^{3}$ Department of Surgery and Translational Medicine, University of Florence, Florence, Italy. \\ ${ }^{4}$ Gastrointestinal Surgery Unit, Florence Careggi University Hospital, Florence, Italy. ${ }^{5}$ Institute of Pathology, Medical University \\ of Graz, Graz, Austria. ${ }^{6}$ Gastro-Intestinal Viral Oncology Group, Ingham Institute for Applied Medical Research, Liverpool, \\ Sydney, New South Wales, Australia. ${ }^{7}$ South Western Sydney Clinical School, University of New South Wales, Kensington, \\ Sydney, New South Wales, Australia. ${ }^{8}$ Department of Gastroenterology \& Hepatology, Bankstown-Lidcombe Hospital, South \\ Western Sydney Local Health Network, Bankstown, Sydney, New South Wales, Australia. ${ }^{9}$ Institute of Pathology, University of \\ Bern, Bern, Switzerland. ${ }^{10}$ Institute of Pathology, Kantonsspital Baselland, Liestal, Switzerland. ${ }^{11}$ Cambridge University \\ Hospitals, NHS Foundation Trust, Cambridge, UK. ${ }^{12}$ Department of Pathology and laboratory Medicine, Cedars-Sinai Medical \\ Center, Los Angeles, California. ${ }^{13}$ Department of Pathology and Laboratory Medicine, Mount Sinai Hospital, Toronto, Ontario, \\ Canada
}

Address for correspondence: Hala El-Zimaity, M.D., Dynacare Laboratory and Health Services Centre, 115 Midair Ct, Brampton, ON L6T 5M3, Canada. hzimaity@gmail.com

Risk factors for esophageal cancer include genetic factors (such as tylosis) and infectious agents. A variety of organisms have been implicated in esophageal carcinogenesis, either directly or indirectly. In this review, we explore the normal esophageal flora and how it may be controlled, and also the variety of organisms that may affect esophageal carcinogenesis, either directly or indirectly. The organisms with potential direct effects in squamous cell carcinoma include human papillomavirus (HPV), Epstein-Barr virus, and polyoma viruses. Interestingly, HPV is now implicated in esophageal adenocarcinoma (EAC), not in its initiation but in the development of dysplasia, in which HPV33 in particular has been associated. Indirectly, Helicobacter pylori has been associated with EAC by, initially, causing increased acid secretion that increases acid reflux, and by reducing lower esophageal sphincter pressure, which increases gastroesophageal reflux; the latter increases the risk of Barrett's esophagus, and hence EAC. Conversely, subsequent atrophic gastritis may normalize that risk.

Keywords: esophagus; carcinoma; infection; microbiota

\section{Introduction}

In looking at the role of infection in esophageal carcinogenesis, it is important to first establish the nature of the normal flora dwelling in all parts of the esophagus and determine if, or how, they change. It is then necessary to look at infectious organisms that may or may not be normally found and determine if they have a role in esophageal carcinogenesis and what the strength of any potential association is. In addition, flora and potential pathogens from adjacent organs may also affect the risk of esophageal carcinoma, since oral organisms such as human papillomavirus (HPV), and potentially other viruses such as Epstein-Barr virus (EBV), can reach the esophagus, either directly in saliva or indirectly systemically, to play a role in esophageal carcinogenesis. Conversely, gastric Helicobacter infection can affect both gastric acid production and the lower esophageal sphincter; so it may contribute to both gastric acid levels as well as gastric contents that may be refluxed into the esophagus, which includes activated pancreaticobiliary secretions and enteric organisms reaching 
the stomach by duodenogastric reflux. We will end by exploring genetic factors such as tylosis and the role of Barrett's esophagus (BE) in esophageal carcinoma.

\section{The normal esophageal microbiota (EM) and its role in esophageal diseases}

\section{The human microbiota}

It is now well recognized that the human microbiota (HM), defined as the complex and dynamic ecosystem that includes trillions of bacteria, archaea, fungi, and viruses with which the human body interacts with in symbiotic and mutualistic relationships, plays a central role in shaping host physiology and health. ${ }^{1}$ Due to its products and its interaction with the host, the HM has been also referred to as an additional organ, even a "second genome", since the HM genetic content (collectively termed microbiome) is more than 100-fold larger than the human genome. ${ }^{2}$ Research in this area has been greatly catalyzed by the recent technological advancements of multiomic sciences, particularly of high-throughput culture-independent metagenomic approaches, which have greatly contributed to expanding our knowledge of the content, diversity, and functions of the $\mathrm{HM}^{3}$

In the last decade, large-scale projects such as the MetaHIT ${ }^{4}$ and the Human Microbiome Project ${ }^{2}$ have provided an exhaustive description of the complex interplay between the HM and host. For example, the gut microbiota (the human body's densest microbial habitat) has an impact on human physiology by participating in the biosynthesis of vitamins and essential amino acids, ${ }^{5}$ by training and modulating the immune system, ${ }^{6}$ and by protecting against epithelial injuries and invading pathogens. $^{7}$

Advancements from these projects have also contributed to identify some of the general features of the HM. First, the HM shows substantial qualitative and quantitative variations through the body axes and among individuals, with thousands of different species having a specific spatial distribution ${ }^{8,9}$ and being unculturable in most cases. Second, important age-dependent compositional variations have been observed over the human life span, with a stable, high level of microbial richness in adulthood, and a lower level in infants and elderly individuals. ${ }^{10}$ Third, the HM is a highly dynamic ecosystem, being able to restore its original healthy equilibrium (a phenomenon known as resilience) following a perturbation of its normal structure (dysbiosis). A common feature of dysbiotic states is a marked decrease of overall microbial diversity, which can be triggered by a plethora of different factors such as infections, antibiotic treatment, and other environmental factors. ${ }^{11,12}$ These microbial imbalances were found to be associated with a number of human pathological conditions. Dysbiotic states have been linked to obesity, inflammatory bowel diseases, Crohn's disease (CD), type II diabetes, liver diseases, bacterial infections, and many other pathological states. ${ }^{73-16}$ However, apart from the fundamental role of gut microbiota in preventing colonization and infection by spore-forming toxigenic strains of Clostridium difficile, ${ }^{17}$ causative relationships between dysbiosis and disease remain to be established.

\section{The EM and its potential role in major esophageal diseases}

Historically, the esophagus was thought to be a relatively sterile organ due to its anatomical and physiological characteristics. The first studies investigating the microbiological content of the esophagus were performed using culture-dependent methods in the 1980s. ${ }^{18,19}$ The few observed organisms, mainly of genus Streptococccus, were supposed to be derived from the oral cavity following the transient passage of saliva and food. It was only in 2004 that Pei and Osias, through microscopic examinations of stained biopsies, clearly showed that the presence of bacteria had an intimate association with the esophageal mucosa. ${ }^{20}$ These findings provided strong evidence for a resident microbiota colonizing the esophagus, as observed in other gastrointestinal tracts, and paved the way for subsequent studies aimed at defining the composition and structure of the microbiota in the normal esophagus and its potential role in the onset or outcome of many esophageal diseases.

Information about healthy EM remains limited, with very few studies enrolling healthy asymptomatic subjects and using culture-independent techniques. Previous findings ${ }^{21}$ were confirmed by recent studies based on next-generation sequencing (NGS), ${ }^{22}$ which demonstrated that the healthy esophagus is dominated by the genus Streptococcus (phylum Firmicutes), in association with Prevotella (phylum Bacteroidetes) and Veillonella (phylum Firmicutes), although at less abundance. Other 
Table 1. Most frequently detected microorganisms at the phylum and genus taxonomic levels ${ }^{a}$

\begin{tabular}{lll}
\hline Phylum & \multicolumn{1}{c}{ Genus } & \multicolumn{1}{c}{ Frequency } \\
\hline Firmicutes & Streptococcus & ++++++++ \\
& Veillonella & ++++ \\
& Granulicatella & ++ \\
Bacteroidetes & Prevotella & ++++ \\
& Bacteroides & ++ \\
& Porphyromonas & +++ \\
Proteobacteria & Haemophilus & ++ \\
& Neisseria & ++ \\
Actinobacteria & Pasteurella & + \\
Fusobacteria & Actinomyces & + \\
\hline & Fusobacterium & +++
\end{tabular}

${ }^{a}$ According to the results of NGS-based studies ${ }^{41,50}$ investigating normal esophageal microbiota.

frequently identified genera included Fusobacterium (phylum Fusobacteria), Haemophilus, Neisseria (phylum Proteobacteria), and Porphyromonas (phylum Bacteroidetes) (Table 1), reinforcing the notion that the esophageal microenvironment is phylogenetically closely related to that found in the oropharynx. $^{23}$

The potential role of EM in participating in disease initiation or promotion has been more thoroughly investigated in relation to gastroesophageal reflux, since it is the strongest modifiable factor involved in the pathogenesis of almost all esophageal diseases. ${ }^{24}$ Prolonged exposure to gastric acid and refluxate is known to severely affect the esophageal mucosa and could elicit marked alterations in microbial communities residing in the lower esophagus. Although studies on the esophageal microenvironment are largely restricted by the need for invasive procedures for sampling, some microbial signatures have been identified as potential contributors to the pathogenesis of gastroesophageal reflux disease (GERD), BE, and esophageal adenocarcinoma (EAC).

A pioneering large-scale culture-independent study, performed by Yang et al. in 2009, ${ }^{25}$ identified two distinct patterns of EM: type I, associated with normal esophagus and characterized by a predominance of Gram-positive taxa, with a high abundance of the genus Streptococcus (79\%); and type II, associated with erosive esophagitis and BE, and characterized by a predominance of Gram-negative taxa (mostly from Bacteroidetes, Fusobacteria, and
Proteobacteria phyla), with a marked reduction in the Streptococcus abundance (30\%). Similar findings were reported in a subsequent study by Liu et al., ${ }^{26}$ whereas recognition of the type I and II patterns was not confirmed by most recent studies employing NGS methodologies, ${ }^{22,27}$ possibly due to differences in patient characteristics (i.e., symptomatic controls, treatment with proton pump inhibitors).

Most recently, Gall et al. analyzed the composition of the EM in a cohort of patients with $\mathrm{BE}$ and identified positive and negative correlations for the Streptococcus-Prevotella ratio with waist-to-hip ratios and hiatal hernia length, respectively, two known risk factors for $\mathrm{BE}$ and EAC. ${ }^{28}$ The incidence of EAC has been rapidly increasing over the past two decades in Western countries, ${ }^{29}$ but only a few culture-independent studies have investigated the potential role of EM in the progression to esophageal malignancies. ${ }^{22,30}$ Blackett et al. reported a significant enrichment of Campylobacter concisus in the early stages of the EAC cascade only (i.e., in GERD and BE patients); they proposed a specific role for this pathogen in the BE/EAC cascade. The proposed pathogenic mechanism consisted in an increase of the host inflammatory response triggered by the chronic colonization by C. concisus. The authors hypothesized that this condition could eventually induce metaplastic changes in a dysbiotic and previously injured esophageal mucosa (i.e., columnar metaplasia characteristic of BE), ultimately favoring malignant transformation. ${ }^{30}$ The most recent study on this topic ${ }^{22}$ did not confirm an enrichment of any Campylobacter species in EAC patients, but instead showed decreased bacterial richness and diversity compared to controls and BE patients, along with a significant increase of Lactobacillus fermentum and lactic acid bacteria in almost half $(47 \%)$ of EAC patients. Moreover, the authors did not identify any differences between the microbiota of BE and EAC patients and suggested that bacterial taxa with very low relative abundances, being more difficult to detect, may potentially contribute to alteration of the EM in these patients. ${ }^{22}$

Overall, current data on EM in health and disease remain limited and a significant gap in knowledge persists about the primary role of EM in disease onset and outcome. Although several hypotheses have suggested how a dysbiotic EM could promote chronic low-grade inflammation and/or favor a chronic reflux state by relaxing 
the lower esophageal sphincter in GERD-related diseases, causation is uncertain. Of note, in addition to GERD-related diseases, compositional variations of the EM have been implicated in less common esophageal diseases and malignancies. ${ }^{31}$

Several issues remain. Little information is available about the stability of EM over time and about any homogeneity of the EM throughout the esophagus. Rare studies have investigated the latter and evaluated upper and lower esophageal biopsy samples in healthy subjects, ${ }^{21}$ normal squamous and metaplastic mucosa in $\mathrm{BE}$ patients, ${ }^{28}$ or normal squamous and malignant mucosa in EAC patients. ${ }^{22}$ In all reported cases, the authors did not identify significant differences between various esophageal segments or histotypes.

Long-term longitudinal studies in a large cohort of patients are therefore needed to answer some of the open questions. Future studies into the EM will be prompted by (1) the formulation of guidelines to cope with the heterogeneous patient enrollment criteria and sampling procedures; (2) the implementation of multiomic approaches; and (3) the development of minimally invasive sampling devices, ${ }^{22}$ which have proven to be as effective as esophageal biopsies in capturing the global diversity observed among esophageal microbial communities.

\section{The role of Helicobacter in EAC}

Since the increase in EAC incidence in Western countries has paralleled the decrease in Helicobacter pylori infection, some investigators suggested Helicobacter protects against EAC. ${ }^{32-34}$ This inverse relationship is frequently attributed to increased gastric acid production following the eradication of $H$. pylori infection. The protective effect is attributed to decreased gastric acid production secondary to H. pylori-associated infection, particularly atrophic pangastritis. ${ }^{32,35,36}$ This protection is primarily seen with cag A-positive strains. ${ }^{37,38}$ Conversely, others have argued that $H$. pylori might be the cause of GERD, ${ }^{39}$ since the infection adversely affects the lower esophageal sphincter. ${ }^{40}$

In practice, the seemingly protective effect of $H$. pylori on GERD has not been seen in countries such as Greece and Turkey, where H. pylori is highly prevalent. ${ }^{41}$ In Turkey, $H$. pylori has no effect on the prevalence of GERD, although it was better controlled by its eradication. ${ }^{41} H$. pylori seems consis- tently protective against GERD in studies conducted in the United States and a few studies from Japan, but inconsistently in studies conducted outside of the United States or Japan. ${ }^{42}$

Chronic H. pylori infection causes either hypoor hyperchlorhydria, depending upon anatomic site of infection (gastritis pattern). Antral-predominant gastritis is associated with hypergastrinemia and hyperchlorhydria and the pattern typically associated with the highest risk of GERD. ${ }^{39,43,44}$ Pangastritis with corpus atrophy, a pattern characteristic of protection, is also highly associated with gastric cancer. ${ }^{44,45}$

Finally, if $H$. pylori is protective against GERD, its eradication would cause reflux; yet studies examining this hypothesis have shown no effect ${ }^{46}$ or GERD symptom improvement following eradication therapy. ${ }^{47}$ Of note, H. pylori eradication is usually followed by a significant increase in body weight. $^{48}$

Though the time trends seem consistent with the hypothesis that declining $H$. pylori prevalence has led to a rise in $\mathrm{BE}$ and increased prevalence of EAC, these trends could be coincidental and not causally related. The increased prevalence of $\mathrm{BE}$ and $\mathrm{EAC}$ could also be related to the higher prevalence of obesity and a decrease in lower esophageal sphincter pressure leading to GERD. ${ }^{49,50}$ It is also possible that both processes may be at work.

\section{The role of HPV infection and HPV vaccines in esophageal squamous cell carcinoma}

Esophageal squamous cell carcinoma (ESCC) accounts for a majority of esophageal cancer occurring most commonly in the upper two-thirds of the esophagus. Rare variants of ESCC include: basaloid squamous cell carcinoma (BSCC), spindle cell (squamous) carcinoma (carcinosarcoma), undifferentiated carcinoma, verrucous (squamous) carcinoma, and carcinoma cuniculatum. ${ }^{51-54}$

Incidence of ESCC shows wide geographical variations, ranging from $2.5 / 100,000$ to $250 / 100,000$ in low- (e.g., the United States, Australia) and high-incidence (e.g., Iran, China, Brazil) countries, respectively. ${ }^{55,56}$ The etiological role of HPV in the carcinogenesis leading to ESCC is still unclear. To date, great differences in HPV DNA detection rates in cancerous tissue have been reported, with a prevalence ranging from $1 \%$ to $65 \%$ within 
the same region and from $0 \%$ to $70 \%$ between different regions. ${ }^{56-59}$ Two different meta-analyses showed significant association between HPV infection and ESCC, with odds ratios of 3.32 and 3.11. 57,60 Furthermore, case-control studies showed highly divergent detection rates of HPV DNA in normal esophagus (range: $0-58.9 \%){ }^{57}$

Over the years, different HPV detection methods with variable specificity and sensitivity have been applied. ${ }^{61}$ Today, in situ hybridization (ISH) and polymerase chain reaction (PCR) are most frequently used, with more reliable results. Nevertheless, one should keep in mind that more than 100 HPV strains have been discovered so far. The oncogenic types are divided into high- and low-risk subtypes based on cervical, oropharyngeal, and anal squamous cell carcinoma. In studies focused on ESCC, only certain strains of HPV (usually type 11, 16 , and 18) have been tested, leaving the possibility of undetected infection by a different HPV type not included in the test. According to published findings, types 16 and 18 are most frequently observed, followed by types $11,31,33$, and $52 .{ }^{57-60}$

In cervical cancer, p16 overexpression is frequently used as evidence of HPV oncoprotein activity. In ESCC, however, studies have not been able to confirm p16 as a reliable marker of transcriptionally active HPV. ${ }^{58,62,63}$ Furthermore, rates of double-positive ESCC (namely HPV-positive and p16-overexpressing) were most commonly found in only about $5 \%$ of carcinomas. ${ }^{58,59}$ Of note, an inconsistent definition of cutoff values for the determination of p16 overexpression was observed among the studies.

Concerning rare subtypes of ESCC, only a small number of studies looking at HPV status have been published in English. Using ISH or PCR, no HPV DNA was detected in nine cases of BSCC, 11 cases of carcinoma cuniculatum, or one case of spindle cell (squamous) carcinoma, suggesting that HPV likely does not play a role in the oncogenesis of these rare variants. ${ }^{53,54,64,65}$ One report suggested an association with HPV type 51 for the development of verrucous carcinoma. The finding of HPV DNA by PCR, however, was not confirmed in a resection specimen from the same patient. ${ }^{66}$

\section{Potential role of vaccines}

Bivalent (HPV-16/18; Cervarix ${ }^{\circledR}$ ) and quadrivalent (HPV-6/11/16/18; Gardasil $\left.{ }^{\circledR}\right)$ vaccines target- ing the most common HPV types have been found to be effective in cervical, vulvar, oral, and anal cancer. ${ }^{67}$ Studies investigating the efficacy of these two vaccines also observed cross-protection against some nonvaccine but oncogenic HPV types (e.g., HPV-31/33/45). More recently, nonavalent (HPV$6 / 11 / 16 / 18 / 31 / 33 / 45 / 52 / 58$; Gardasil $\left.{ }^{\circledR}\right)$ vaccine has been approved in some countries. Unfortunately, studies evaluating the efficacy of HPV vaccines in the oncogenesis of ESCC are lacking. Prophylactic vaccination could be promising and beneficial in the reduction of HPV-associated ESCC in regions with high ESCC incidence, and this effect could be increased in countries where both males and females are vaccinated.

\section{HPV and adenocarcinoma of the esophagus}

GERD and BE have traditionally been considered the most important risk factors for EAC, the fastest growing cancer in the Western world and occurring against a backdrop of progressive reduction in the risk of malignancy associated with $\mathrm{BE}$ but no reduction in mortality from EAC, using the prevailing screening and surveillance guidelines. ${ }^{68}$ The recently published link between high risk HPV and Barrett's dysplasia (BD)/cancer may be one of the missing strong risk factors responsible for the significant rise of EAC since the 1970s, as has been the case with head and neck squamous cell carcinomas, a significant minority of which are also virally associated. ${ }^{69,70}$

Previously published studies using PCR techniques that showed negative results were due to poor tissue classification, suboptimal testing methodology exacerbated by low viral load, old specimen samples susceptible to DNA/RNA degradation, as well as ethnic and regional variations. ${ }^{71-75}$ Instead of fresh frozen material, almost all of these retrospective investigations used paraffin-embedded tissue, which yields a significant false negative HPV PCR DNA result. ${ }^{68}$ Some studies misclassified as negative used only nondysplastic BE. Barrett's metaplastic tissue has never been shown to be HPV-associated. ${ }^{71,72}$ Others have detected only lowrisk HPV types without seeking evidence of viral integration or replication. ${ }^{76}$ Interestingly, a systematic review reported HPV prevalence rates of $35 \%$ in 174 cases of EAC, not dissimilar to other findings. ${ }^{77}$ Another systematic review that included 19 studies 


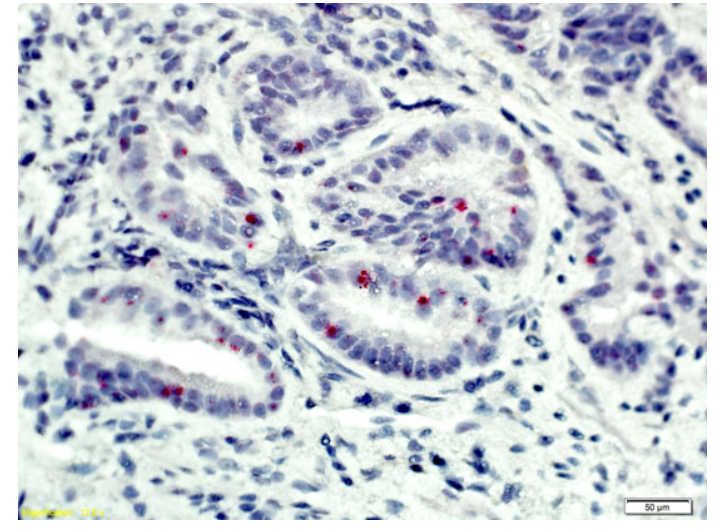

Figure 1. In situ hybridization detection of transcriptional activity of hr-HPV (i.e., E6/E7 mRNA) in esophageal adenocarcinoma. Positive staining is diffusely present (with a punctate/granular appearance) in the nuclei and cytoplasm (image courtesy of Professor S. Rajendra).

concluded that the pooled prevalence of HPV in EAC was $13 \%$. The authors suggested that the low prevalence rate may have been caused by small sample sizes and compromised detection methods. ${ }^{78}$ An important reason for why it is difficult to detect HPV DNA via PCR is the low viral load in glandular tissue ${ }^{79}$ which is very much less than in the uterine cervix, anal canal, and oropharynx, which all consist of squamous epithelium. It is well known that HPV has a predilection for squamous epithelium, though ISH analysis of HPV DNA and E6/E7 mRNA has clearly demonstrated viral tropism for esophageal glandular tissue (Figs. 1 and 2).

In 2013, one study demonstrated that biologically active, high-risk HPV (hr-HPV) is strongly associated with $\mathrm{BD}$ and EAC. ${ }^{70,79}$ Viral-host genome integration was found to be an early event. ${ }^{79}$ An increasing HPV viral load and integration gradient was significantly linked with disease severity as per the Barrett's metaplasia-dysplasia-adenocarcinoma pathway. ${ }^{79}$ Moreover, persistent hr-HPV infection and p53 overexpression predicted treatment failure after endoscopic ablation of BD and EAC. ${ }^{80}$ The vast majority of $\mathrm{BD}$ and $\mathrm{EAC}$ specimens containing biologically active hr-HPV were shown to have wild-type TP53, which is typical of HPV-driven cancers. ${ }^{60,68,81}$ Recently, it was demonstrated that $\mathrm{HPV}$-associated EAC harbors a distinct profile of molecular aberrations/genomic abnormalities compared with HPV-negative EAC, indicating differ-

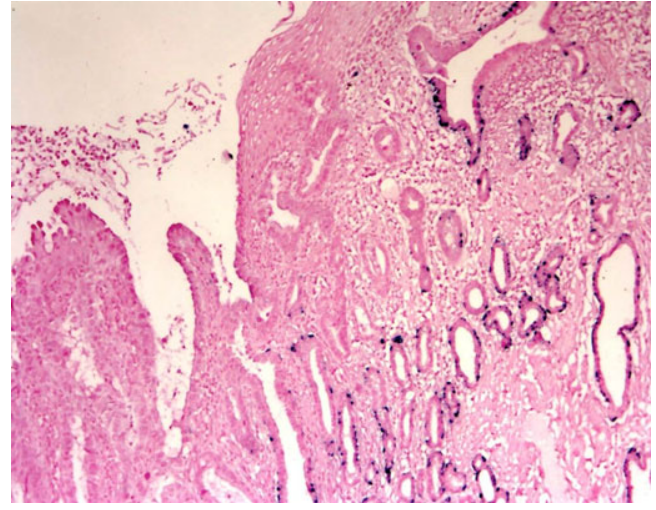

Figure 2. DNA in situ hybridization image revealing the presence of high-risk human papillomavirus genome in a Barrett's dysplasia/esophageal adenocarcinoma specimen. Absence of staining in adjacent squamous epithelium suggests viral tropism for esophageal glandular tissue (image courtesy of Professor S. Rajendra).

ent biological mechanisms of tumor formation. ${ }^{82}$ HPV-positive EAC was shown to have half the number of nonsilent somatic mutations than HPVnegative esophageal cancers. TP53 aberrations were absent in the HPV-positive EAC group, whereas $50 \%$ of the HPV-negative EAC patients exhibited TP53 mutations. Viral integration analysis identified hybrid sequences containing HPV16 and the human genome, providing evidence for a hostviral interaction. ${ }^{82}$ Active hr-HPV involvement in $\mathrm{BD} / \mathrm{EAC}$ is identified by a characteristic molecular signature consisting of retinoblastoma protein (degraded by the E7 oncoprotein) pathway alterations, for example, upregulation of $\mathrm{p} 16^{\mathrm{INK} 4 \mathrm{~A}}$ as well as downregulation of $\mathrm{pRb}$ wild-type and p53 (the target of the E6 viral cancer protein; Fig. 1). ${ }^{83}$ Thus, hr-HPV is considered a risk factor for BD and EAC (in $20-25 \%$ of patients), along with chronic reflux, obesity, and smoking. ${ }^{84}$

\section{The role of EBV in esophageal cancer}

\section{Biological background of EBV infection in gastrointestinal carcinomas and the role of EBV in esophageal carcinomas}

EBV is one of the nine known viruses in the family of human Herpesviridae (HHV-4). These DNA viruses typically show latent, recurrent infectionshence the term herpes, derived from the Greek word herpein (= to creep). EBV affects over 95\% of the adult population. While childhood infection 
with EBV is usually symptomless or indistinguishable from other mild, brief childhood illnesses, its manifestation during adolescence as infectious mononucleosis may present more severe and partly long-lasting symptoms. ${ }^{85,86} \mathrm{EBV}$ is also associated with specific forms of cancer, including both hematologic and epithelial malignancies, and it is estimated that worldwide over 200,000 cancer cases and up to $2 \%$ of cancer-related deaths per year may be attributed to infection with the virus..$^{87,88}$

EBV infection occurs by oral transfer of saliva, and genital secretions. EBV is a lymphotropic virus that infects B lymphocytes via CD21/CR complement receptor type 2. After an initial lytic infection, EBV latency persists in B cells for the rest of the host's life. This direct infection of B cells can later cause suppression of somatic hypermutation after latency, leading to a continuous proliferation of the infected clone eventually resulting in the establishment of a lymphoma. Another possibility for lymphomagenesis is the direct infection of memory B cells, with subsequent induction of proliferation. Lymphomas that are typically associated with EBV are Burkitt lymphoma, classical Hodgkin lymphoma, EBV-positive posttransplantation lymphoma, and some cases of diffuse large B cell lymphomas and $\mathrm{T}$ cell lymphomas. ${ }^{88}$

The majority of EBV-associated cancers, however, are epithelial malignancies, and the most frequent types by far are nasopharyngeal carcinomas and gastric adenocarcinomas. ${ }^{87}$ Epithelial cells lack CD21 as a surface receptor for EBV infection, but the virus may enter epithelial cells using saliva IgA as a vehicle, by the interaction with integrins on polarized epithelial cells or using other virus components as ligands of specific epithelial surface receptors. ${ }^{89}$ There is also evidence of a shuttle between infected B cells releasing EBV and epithelial cells being infected, and vice versa. This cell-to-cell, contactmediated EBV infection is also far more effective compared to the cell-free infection mode of B cell infection..$^{90}$ This also explains the fact that EBV infection of epithelial cells predominantly occurs in anatomic sites with well-established surrounding lymphatic tissue (e.g., the nasopharyngeal tract or mucosa-associated lymphatic tissue) or in association with chronic infections (e.g., chronic gastritis).

On a molecular level, EBV infection causes a large variety of events. ${ }^{91}$ This includes mutations (e.g., PIK3CA, ARID1A, AKT2, and many others), methy- lation of both the EBV and the host genome, amplifications (e.g., JAK2, CD274, PDCD1LG2, and others), deletions (e.g., PTEN, MACROD2, and others), and deregulation of the expression of a large variety of mRNAs and microRNAs (miRNAs)..${ }^{91-93}$ This hypermutated status of EBV-associated carcinomas is the underlying reason for the well-established inflammatory infiltrate that often accompanies these types of cancers, resulting in the typical lymphoepithelial phenotype.

The association of EBV with a subset of gastric adenocarcinomas and adenocarcinomas of the gastroesophageal junction has been well documented over the last few decades, and EBV positivity now also defines a specific molecular subtype of gastric adenocarcinoma that is highly distinct from EBV-negative tumors. ${ }^{94,95}$ The association of EBV with esophageal carcinoma, however, is less clearly defined, as the relatively few studies on this matter show conflicting results. ${ }^{78,96,97}$ These discrepancies can be primarily attributed to the different methods used to detect EBV. Older studies relying on PCR-based methods lack specificity, as EBVpositive lymphocytes are found in the majority of the adult population. Thus, the detection of EBVpositive tumor infiltrating lymphocytes in tumor tissue by PCR results in false positive results for EBV presence in the neoplastic cells. In contrast, direct evidence of EBV in individual tumor cells by ISH or immunohistochemistry is more specific, with the disadvantage of being less sensitive. Studies focusing on the detection of EBV within tumor cells suggest that EBV is not associated with EAC and conventional ESCC. ${ }^{78,98}$ However, the very rare squamous cell carcinoma variant of lymphoepithelial carcinoma or lymphoepithelioma-like carcinoma has been shown to be associated with EBV infection in several studies..$^{97,99-101}$ This frequently EBV-positive tumor is defined as an undifferentiated or poorly differentiated squamous cell carcinoma with a prominent lymphoplasmacytic infiltrate, morphologically similar to nasopharyngeal lymphoepithelial carcinoma. It is more prevalent in Asia, has a distinct submucosal-like appearance on endoscopy, and is associated with an improved outcome in comparison to conventional squamous cell carcinoma. ${ }^{99}$ Whether EBV-positive esophageal lymphoepithelial carcinoma features high levels of PD-L1/PD-L2 expression analogously to EBV-positive gastric adenocarcinoma and thus 
might benefit from immune checkpoint inhibition is currently unknown. ${ }^{94}$

\section{Polyomavirus and esophageal carcinogenesis}

Polyomavirus $(\mathrm{PyV})$ is a nonenveloped doublestranded DNA virus that infects birds, mammals, and fish. There are 73 recognized species, of which 13 infect human hosts and are grouped together as the human polyomaviruses (HPyVs) ${ }^{102} \mathrm{HPyVs}$ are highly prevalent in aqueous environments, including urban sewage, and have been isolated from different surface water samples. ${ }^{103}$ They can persist as latent infection for a long time without causing disease in hosts. The genome of most PyVs consists of approximately 5000 base-pairs and encodes regulatory (large and small $\mathrm{T}$ antigen) and structural capsid proteins (VP1, VP2). Some HPyVs also have an additional regulatory protein, agnoprotein. ${ }^{104}$ $\mathrm{BK}$ virus (BKV) and the JC virus (JCV) were the first two HPyVs isolated from human hosts. The oncogenic potential of these two HPyVs, along with a third common type of $\mathrm{HPyV}$, Merkel cell $\mathrm{PyV}$ $(\mathrm{MCPyV})$, is well documented in cell culture and animal model studies. MCPyV has a strong association with cancer in its natural host; approximately $80 \%$ of Merkel cell carcinoma tumors are positive for MCPyV. ${ }^{105} \mathrm{McPyV}$ has also been isolated from tissue samples of normal upper aerodigestive tract, including oral cavity and esophagus. ${ }^{106}$ The etiology of esophageal cancer is multifactorial, and the role of viruses is still controversial. With regard to the role of HPyVs, two different studies have demonstrated a causal association between $\mathrm{McPyV}$ and JCPyV in esophageal carcinogenesis. In a cross-sectional study, Yahyapour et al. analyzed 96 formalin-fixed resection specimens from ESCC and benign conditions. HPV and $\mathrm{McPyV}$ DNA load in tissue samples were measured by using the real-time PCR technique. MCPyV DNA was detected in $45.1 \%$ of esophageal cancer samples and $35.6 \%$ of benign pathology samples. There was no statistically significant difference between the mean MCPyV DNA load $(P=0.353)$ of both sample types. Esophageal samples with normal histology were negative for MCPyV DNA. HPV DNA was detected in $31.4 \%$ of cancer samples and $44.4 \%$ in the benign samples. Concomitant infection with HPV and MCPyV was seen in $15.8 \%$ of cancer and $17.8 \%$ of benign esophageal samples. ${ }^{107}$
This study provides some evidence for $\mathrm{MCPyV}$ infection in esophageal tissues, either alone or with HPV, however, an unequivocal contribution to esophageal carcinogenesis is debatable; more extensive studies are needed.

\section{JCV in esophageal carcinoma}

A subtype of $\mathrm{HPyV}, \mathrm{JCV}$ is a well-recognized causative agent of progressive multifocal leukoencephalopathy in immunocompromised hosts. ${ }^{108}$ Subclinical infection in humans is known to be present in $85-90 \%$ of the population worldwide. In addition to early and late coding genes, the JCV genome also contains the small regulatory protein agnoprotein. Its oncogenic potential has been studied in experimental animals and is associated with human brain tumors. JCV has also been found in normal mucosa of the gastrointestinal tract. ${ }^{8}$ T-antigen protein of JCV has been isolated from colon carcinomas. ${ }^{109,110}$

In a study performed using immunohistochemical and PCR techniques, Del Valle et al. demonstrated the presence of JCV DNA sequences and proteins in normal, benign, and cancer pathology tissue samples. Esophageal tissue biopsy samples from 70 subjects, including normal esophagus, a varied range of benign conditions, adenocarcinoma, and squamous cell carcinoma were studied. JCV DNA sequences were found in cells from normal esophagus, benign esophageal diseases, and esophageal carcinoma. Notably, expression of active viral proteins, including $\mathrm{T}$ antigen and agnoprotein, was detected in many of the cancer samples. ${ }^{111}$ The oncogenic potential of these proteins might have contributed to the carcinogenesis in this study. ${ }^{12}$ Conclusive proof of their specific role in esophageal carcinogenesis is lacking and further studies are warranted.

\section{Esophageal carcinoma in tylosis}

Tylosis (hyperkeratosis palmaris et plantaris) is a rare inherited condition characterized by focal nonepidermolytic thickening of the skin in the palms and soles. ${ }^{13,114}$ Tylosis with esophageal cancer (TOC) was first described in two British families (Liverpool, the United Kingdom). ${ }^{15,116}$ Tylosis is associated with a very high lifetime risk of developing squamous cell carcinoma of the esophagus: $95 \%$ at the age of 65 in one Liverpool family. ${ }^{115}$ Its clinical aspects, diagnosis, and management have been reviewed by Ellis et al. ${ }^{117}$ 


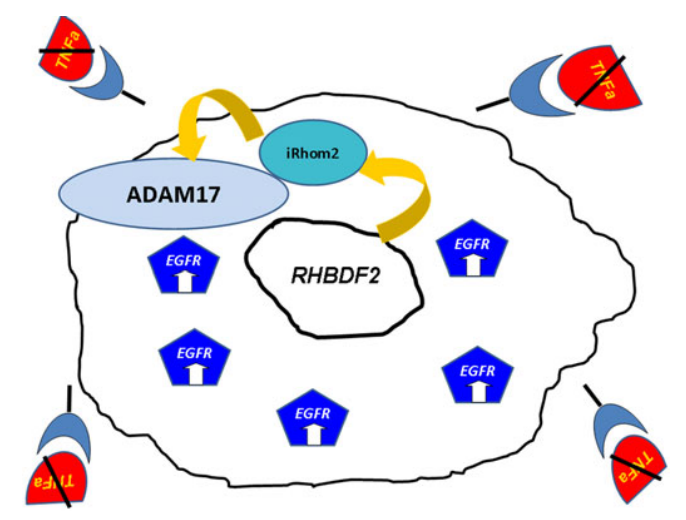

Figure 3. Keratinocyte/squamous cell with a mutated RHBDF2 gene (in the nucleus) that encodes an inactive rhomboid protein, leading to (1) increased shedding of ADAM17 substrates, including EGF-family growth factors, and increased EGFR activity; and (2) shedding of TNF receptors from cell surfaces, muting the cellular response to TNF- $\alpha$.

It is inherited as an autosomal dominant trait with complete penetrance. Missense mutations have been described in rhomboid family member 2 (RHBDF2) located on 17q25.1, which encodes an inactive rhomboid protein 2 (iRhom 2). ${ }^{118}$ RHBDF2 is involved in the regulation of the secretion of several ligands for the epidermal growth factor receptor. Mutations in RHBDF2 inhibit tumor necrosis factor alpha (TNF- $\alpha$; i.e., dampens its cell response) and enhance epidermal growth factor receptor (EGFR) activity. ${ }^{119}$

TOC-associated iRHOM2 mutations increase the maturation and activity of ADAM metallopeptidase domain 17 (ADAM17), a protease that catalyzes the shedding of various transmembrane proteins from the surface of cells, which leads to a significant increase in the shedding of ADAM17 substrates from epidermal keratinocytes, including EGF-family growth factors, and increased EGFR activity. These features are consistent with the presence of a constitutive wound-healing-like phenotype in TOC epidermis (Fig. 3). ${ }^{119}$

ADAM17 also catalyzes the shedding of TNF- $\alpha$ and its receptors. Liberation of TNF receptors from cell surfaces can dampen the cellular response to TNF- $\alpha$, a cytokine that promotes programmed cell death (Fig. 1).

Deletions in the cytoplasmic domain of iRhom1 and iRhom 2 promote shedding of the TNF receptor by ADAM17. ${ }^{120}$ Mutated iRhom 2 increases ADAM17 activity, TNF receptor shedding, and resistance to TNF-induced cell death in fibrosarcoma cells. Keratinocytes from TOC patients exhibit increased TNF receptor shedding compared with cells from healthy donors. It appears that aberrant EGFR signaling underlies the predisposition for ESCC.

\section{Role of BE in ESCC}

Squamous carcinoma has been described in patients with $\mathrm{BE}$ in a variety of settings. There are multiple case reports in the literature describing synchronous squamous cell carcinoma and adenocarcinoma in the esophagus with Barrett's mucosa. ${ }^{121-123}$ In these reports, the squamous cell carcinoma did not arise within the Barrett's segment.

A study of esophagectomy specimens with squamous cell carcinoma and adenocarcinoma found Barrett's mucosa (without or with dysplasia) in $100 \%$ of specimens with an adenocarcinoma and in $40.6 \%$ with squamous cell carcinoma. ${ }^{123}$ Highgrade squamous dysplasia was present in 10 of the 13 specimens with squamous cell carcinoma, adjacent to the squamous tumor. The squamous cell carcinoma and/or high-grade squamous dysplasia were separated from the Barrett's mucosa by normal squamous epithelium.

Barrett's adenocarcinoma and squamous cell carcinoma share some risk factors in common: age, male gender, and smoking. ${ }^{124,125}$ The latter is somewhat controversial in the literature, but there are reports of a causal relationship between smoking and Barrett's adenocarcinoma. ${ }^{126}$

Squamous carcinoma may be seen in $\mathrm{BE}$ as a component of adenosquamous carcinoma in which the glandular and squamous components are intimately admixed. Organ sites that develop adenocarcinoma can also develop adenosquamous carcinoma $^{127}$ and this is thought to represent tumor transdifferentiation. There are case reports of adenosquamous carcinoma that support this contention. Mishima et al. described an adenocarcinoma occurring in Barrett's mucosa where histological examination showed the focal squamous cell carcinoma component present within the adenocarcinoma. ${ }^{128}$ The Barrett's mucosa surrounding the adenocarcinoma component of the tumor displayed glandular dysplasia as a precursor to the adenocarcinoma.

Van Rees et al. found molecular evidence for the monoclonal origin of both components of 
adenosquamous carcinoma in Barrett's mucosa. ${ }^{129}$ The Barrett's epithelium, the adenocarcinoma, and the squamous cell carcinoma shared the same allelic loss on chromosome 9p, and the adenocarcinoma and the squamous cell carcinoma had the same allelic loss on chromosomal arms 3p, 5q, 10q, $14 \mathrm{q}$, and 18q. Sequencing of the p53 gene revealed the same missense mutation in the adenocarcinoma and the squamous cell carcinoma component.

Squamous islands occur in Barrett's mucosa, whether untreated ${ }^{130}$ or treated after acid suppression, ${ }^{131,132}$ where they may be related to Barrett's epithelium regression. These squamous cells situated within the Barrett's segment could potentially serve as a source of squamous cell carcinoma development. A study analyzing tumor biomarkers (Ki-67 and p53) suggests that completely reversed squamous epithelium following a combination of thermal and proton pump inhibitor therapy is biologically similar to normal squamous epithelium and is of low cancer risk, whereas partially reversed squamous epithelium manifested as squamous islands is accompanied by biomarker abnormalities suggesting a continued cancer risk. ${ }^{133}$

There are case reports of the occurrence of squamous cell carcinoma after endoscopic mucosal ablation $^{134}$ and radiofrequency ablation (RFA), used as therapy for Barrett's mucosa. In the first report of squamous cell carcinoma after RFA, Barrett's mucosa with high-grade dysplasia initially responded well to RFA with the development of neosquamous epithelium. ${ }^{135}$ However, squamous cell carcinoma rapidly developed. Sanger sequencing confirmed that the original high-grade dysplasia and the squamous cell carcinoma had separate clonal origins. The rapid development after RFA and occurrence of the squamous tumor at the same location as the high-grade dysplasia strongly argue in favor of causal relationship with the RFA.

To summarize, BE can predispose to, or be associated with, squamous cell carcinoma by several mechanisms (1) shared risk factors that give rise to squamous and adenocarcinoma as separate tumors, possibly synchronous; (2) squamous cell carcinoma occurring as a component in a Barrett's adenocarcinoma, likely representing divergent or tumor transdifferentiation; and (3) as a consequence of medical or local ablative therapies for Barrett's mucosa.
Scenarios 2 and 3 can be considered as predisposing mechanisms.

\section{Competing interests}

The authors declare no competing interests.

\section{References}

1. Lloyd-Price, J., G. Abu-Ali \& C. Huttenhower. 2016. The healthy human microbiome. Genome Med. 8: 51.

2. Human Microbiome Project Consortium. 2012. Structure, function and diversity of the healthy human microbiome. Nature 486: 207-214.

3. Jansson, J.K. \& E.S. Baker. 2016. A multi-omic future for microbiome studies. Nat. Microbiol. 1: 16049.

4. Qin, J., R. Li, J. Raes, et al. 2010. A human gut microbial gene catalogue established by metagenomic sequencing. Nature 464: 59-65.

5. Sommer, F. \& F. Backhed. 2013. The gut microbiotamasters of host development and physiology. Nat. Rev. Microbiol. 11: 227-238.

6. Sonnenberg, G.F. \& D. Artis. 2012. Innate lymphoid cell interactions with microbiota: implications for intestinal health and disease. Immunity 37: 601-610.

7. Lynch, S.V. \& O. Pedersen. 2016. The human intestinal microbiome in health and disease. N. Engl. J. Med. 375: 2369-2379.

8. Group, N.H.W., J. Peterson, S. Garges, et al. 2009. The NIH Human Microbiome Project. Genome Res. 19: 2317-2323.

9. Donaldson, G.P., S.M. Lee \& S.K. Mazmanian. 2016. Gut biogeography of the bacterial microbiota. Nat. Rev. Microbiol. 14: $20-32$.

10. Saraswati, S. \& R. Sitaraman. 2014. Aging and the human gut microbiota-from correlation to causality. Front. Microbiol. 5: 764.

11. Greenhalgh, K., K.M. Meyer, K.M. Aagaard, et al. 2016. The human gut microbiome in health: establishment and resilience of microbiota over a lifetime. Environ. Microbiol. 18: $2103-2116$.

12. Sommer, F., J.M. Anderson, R. Bharti, et al. 2017. The resilience of the intestinal microbiota influences health and disease. Nat. Rev. Microbiol. 15: 630-638.

13. Omer, E. \& H. Atassi. 2017. The microbiome that shapes us: can it cause obesity? Curr. Gastroenterol. Rep. 19: 59.

14. Zhang, M., K. Sun, Y. Wu, et al. 2017. Interactions between intestinal microbiota and host immune response in inflammatory bowel disease. Front. Immunol. 8: 942.

15. Shen, Z., C. Zhu, Y. Quan, et al. 2017. Update on intestinal microbiota in Crohn's disease 2017: mechanisms, clinical application, adverse reactions, and outlook. J. Gastroenterol. Hepatol. 32: 1804-1812.

16. Bhatt, A.P., M.R. Redinbo \& S.J. Bultman. 2017. The role of the microbiome in cancer development and therapy. $C A$ Cancer J. Clin. 67: 326-344.

17. Seekatz, A.M. \& V.B. Young. 2014. Clostridium difficile and the microbiota. J. Clin. Invest. 124: 4182-4189.

18. Lau, W.F., J. Wong, K.H. Lam, et al. 1981. Oesophageal microbial flora in carcinoma of the oesophagus. Aust. N. Z. J. Surg. 51: 52-55. 
19. Mannell, A., M. Plant \& J. Frolich. 1983. The microflora of the oesophagus. Ann. R. Coll. Surg. Engl. 65: 152-154.

20. Osias, G.L., M.Q. Bromer, R.M. Thomas, et al. 2004. Esophageal bacteria and Barrett's esophagus: a preliminary report. Dig. Dis. Sci. 49: 228-236.

21. Norder Grusell, E., G. Dahlen, M. Ruth, et al. 2013. Bacterial flora of the human oral cavity, and the upper and lower esophagus. Dis. Esophagus 26: 84-90.

22. Elliott, D.R.F., A.W. Walker, M. O’Donovan, et al. 2017. A non-endoscopic device to sample the oesophageal microbiota: a case-control study. Lancet Gastroenterol. Hepatol. 2: 32-42.

23. Arweiler, N.B. \& L. Netuschil. 2016. The oral microbiota. Adv. Exp. Med. Biol. 902: 45-60.

24. Tack, J. \& J.E. Pandolfino. 2018. Pathophysiology of gastroesophageal reflux disease. Gastroenterology 154: 277-288.

25. Yang, L., X. Lu, C.W. Nossa, et al. 2009. Inflammation and intestinal metaplasia of the distal esophagus are associated with alterations in the microbiome. Gastroenterology 137: 588-597.

26. Liu, N., T. Ando, K. Ishiguro, et al. 2013. Characterization of bacterial biota in the distal esophagus of Japanese patients with reflux esophagitis and Barrett's esophagus. BMC Infect. Dis. 13: 130.

27. Amir, I., F.M. Konikoff, M. Oppenheim, et al. 2014. Gastric microbiota is altered in oesophagitis and Barrett's oesophagus and further modified by proton pump inhibitors. Environ. Microbiol. 16: 2905-2914.

28. Gall, A., J. Fero, C. McCoy, et al. 2015. Bacterial composition of the human upper gastrointestinal tract microbiome is dynamic and associated with genomic instability in a Barrett's Esophagus Cohort. PLoS One 10: e0129055.

29. Rustgi, A.K. \& H.B. El-Serag. 2014. Esophageal carcinoma. N. Engl. J. Med. 371: 2499-2509.

30. Blackett, K.L., S.S. Siddhi, S. Cleary, et al. 2013. Oesophageal bacterial biofilm changes in gastro-oesophageal reflux disease, Barrett's and oesophageal carcinoma: association or causality? Aliment Pharmacol. Ther. 37: 1084-1092.

31. Di Pilato, V., G. Freschi, M.N. Ringressi, et al. 2016. The esophageal microbiota in health and disease. Ann. N.Y. Acad. Sci. 1381: 21-33.

32. Koike, T., S. Ohara, H. Sekine, et al. 1999. Helicobacter pylori infection inhibits reflux esophagitis by inducing atrophic gastritis. Am. J. Gastroenterol. 94: 3468-3472.

33. de Martel, C., A.E. Llosa, S.M. Farr, et al. 2005. Helicobacter pylori infection and the risk of development of esophageal adenocarcinoma. J. Infect. Dis. 191: 761-767.

34. Anderson, L.A., S.J. Murphy, B.T. Johnston, et al. 2008. Relationship between Helicobacter pylori infection and gastric atrophy and the stages of the oesophageal inflammation, metaplasia, adenocarcinoma sequence: results from the FINBAR case-control study. Gut 57: 734-739.

35. Ye, W., M. Held, J. Lagergren, et al. 2004. Helicobacter pylori infection and gastric atrophy: risk of adenocarcinoma and squamous-cell carcinoma of the esophagus and adenocarcinoma of the gastric cardia. J. Natl. Cancer Inst. 96: 388-396.

36. McColl, K.E., H. Watabe \& M.H. Derakhshan. 2008. Role of gastric atrophy in mediating negative association between
Helicobacter pylori infection and reflux oesophagitis, Barrett's oesophagus and oesophageal adenocarcinoma. Gut 57: 721-723.

37. Chow, W.H., M.J. Blaser, W.J. Blot, et al. 1998. An inverse relation between $\mathrm{cagA}^{+}$strains of Helicobacter pylori infection and risk of esophageal and gastric cardia adenocarcinoma. Cancer Res. 58: 588-590.

38. Islami, F. \& F. Kamangar. 2008. Helicobacter pylori and esophageal cancer risk: a meta-analysis. Cancer Prev. Res. (Phila). 1: 329-338.

39. Tomasello, G., F. Giordano, M. Mazzola, et al. 2017. Helicobacter pylori and Barrett's esophagus: a protective factor or a real cause? J. Biol. Regul. Homeost. Agents 31: 9-15.

40. Rossi, G., R. Gambi, R. Uncini, et al. 2014. Severe gastritis with double Helicobacter spp. infection associated with Barrett's esophagus in a cheetah. Helicobacter 19: 462464.

41. Bor, S., G. Kitapcioglu \& E. Kasap. 2017. Prevalence of gastroesophageal reflux disease in a country with a high occurrence of Helicobacter pylori. World J. Gastroenterol. 23: 525-532.

42. Fischbach, L.A., H. Nordenstedt, J.R. Kramer, et al. 2012. The association between Barrett's esophagus and Helicobacter pylori infection: a meta-analysis. Helicobacter. 17: 163175.

43. Nobakht, H., A. Boghratian, M. Sohrabi, et al. 2016. Association between pattern of gastritis and gastroesophageal reflux disease in patients with Helicobacter pylori infection. Middle East J. Dig. Dis. 8: 206-211.

44. Graham, D.Y. 2003. Helicobacter pylori is not and never was "protective" against anything, including GERD. Dig. Dis. Sci. 48: 629-630.

45. Uemura, N., S. Okamoto, S. Yamamoto, et al. 2001. Helicobacter pylori infection and the development of gastric cancer. N. Engl. J. Med. 345: 784-789.

46. Xue, Y., L.Y.Zhou, S.R. Lin, et al. 2015. Effect of Helicobacter pylori eradication on reflux esophagitis therapy: a multicenter randomized control study. Chin. Med. J. (Engl). 128: 995-999.

47. Moschos, J.M., G. Kouklakis, S. Vradelis, et al. 2014. Patients with established gastro-esophageal reflux disease might benefit from Helicobacter pylori eradication. Ann. Gastroenterol. 27: 352-356.

48. Choi, J.S., K.O. Ko, J.W. Lim, et al. 2016. The association between Helicobacter pylori infection and body weight among children. Pediatr. Gastroenterol. Hepatol. Nutr. 19: 110-115.

49. Tanaka, Y., Y. Sakata, M. Hara, et al. 2017. Risk factors for Helicobacter pylori infection and endoscopic reflux esophagitis in healthy young Japanese volunteers. Intern. Med. 56: 2979-2983.

50. Richter, J.E. \& J.H. Rubenstein. 2018. Presentation and epidemiology of gastroesophageal reflux disease. Gastroenterology. 154: 267-276.

51. Montgomery, E., J.K. Field, P. Boffetta, et al. 2010. Squamous cell carcinoma of the oesophagus. In WHO Classification of Tumours of the Digestive System. F.T. Bosman, F. Carneiro, R.H. Hruban, et al., Eds.: 18-24. Lyon: International Agency for Research on Cancer. 
52. Tripathi, M. \& P.E. Swanson. 2016. Rare tumors of esophageal squamous mucosa. Ann. N.Y. Acad. Sci. 1381: 122-132.

53. De Petris, G., M. Lewin \& T. Shoji. 2005. Carcinoma cuniculatum of the esophagus. Ann. Diagn. Pathol. 9: 134-138.

54. Landau, M., J.R. Goldblum, T. DeRoche, et al. 2012. Esophageal carcinoma cuniculatum: report of 9 cases. Am. J. Surg. Pathol. 36: 8-17.

55. Syrjanen, K.J. 2002. HPV infections and oesophageal cancer. J. Clin. Pathol. 55: 721-728.

56. Hardefeldt, H.A., M.R. Cox \& G.D. Eslick. 2014. Association between human papillomavirus (HPV) and oesophageal squamous cell carcinoma: a meta-analysis. Epidemiol. Infect. 142: 1119-1137.

57. Liyanage, S.S., B. Rahman, I. Ridda, et al. 2013. The aetiological role of human papillomavirus in oesophageal squamous cell carcinoma: a meta-analysis. PLoS One 8: e69238.

58. Michaelsen, S.H., C.G. Larsen \& C. von Buchwald. 2014. Human papillomavirus shows highly variable prevalence in esophageal squamous cell carcinoma and no significant correlation to p16INK4a overexpression: a systematic review. J. Thorac. Oncol. 9: 865-871.

59. Ludmir, E.B., S.J. Stephens, M. Palta, et al. 2015. Human papillomavirus tumor infection in esophageal squamous cell carcinoma. J. Gastrointest. Oncol. 6: 287-295.

60. Ojesina, A.I., L. Lichtenstein, S.S. Freeman, et al. 2014. Landscape of genomic alterations in cervical carcinomas. Nature 506: 371-375.

61. Petrick, J.L., A.B. Wyss, A.M. Butler, et al. 2014. Prevalence of human papillomavirus among oesophageal squamous cell carcinoma cases: systematic review and meta-analysis. Br. J. Cancer 110: 2369-2377.

62. Wang, L., J. Li, J. Hou, et al. 2016. p53 expression but not p16(INK4A) correlates with human papillomavirusassociated esophageal squamous cell carcinoma in Kazakh population. Infect. Agent Cancer 11: 19.

63. Pastrez, P.R.A., V.S. Mariano, A.M. da Costa, et al. 2017. The relation of HPV infection and expression of p53 and p16 proteins in esophageal squamous cells carcinoma. $J$. Cancer 8: 1062-1070.

64. Bellizzi, A.M., R.L. Woodford, C.A. Moskaluk, et al. 2009. Basaloid squamous cell carcinoma of the esophagus: assessment for high-risk human papillomavirus and related molecular markers. Am. J. Surg. Pathol. 33: 1608-1614.

65. Regragui, A., H. Lakhdar, M. Abderrahman Alaoui Belabbas, et al. 2004. [Esophageal sarcomatoid carcinoma: report of a case with morphological, immunohistochemical and molecular study]. Gastroenterol. Clin. Biol. 28: 487-489.

66. Tonna, J., J.M. Palefsky, J. Rabban, et al. 2010. Esophageal verrucous carcinoma arising from hyperkeratotic plaques associated with human papilloma virus type 51. Dis. Esophagus 23: E17-E20.

67. Skinner, S.R., D. Apter, N. De Carvalho, et al. 2016. Human papillomavirus (HPV)-16/18 AS04-adjuvanted vaccine for the prevention of cervical cancer and HPV-related diseases. Expert Rev. Vaccines 15: 367-387.

68. Rajendra, S. 2015. Barrett's oesophagus: can meaningful screening and surveillance guidelines be formulated based on new data and rejigging the old paradigm? Best Pract. Res. Clin. Gastroenterol. 29: 65-75.

69. Chaturvedi, A.K., E.A. Engels, R.M. Pfeiffer, et al. 2011. Human papillomavirus and rising oropharyngeal cancer incidence in the United States. J. Clin. Oncol. 29: 42944301.

70. Rajendra, S., B. Wang, E.T. Snow, et al. 2013. Transcriptionally active human papillomavirus is strongly associated with Barrett's dysplasia and esophageal adenocarcinoma. Am. J. Gastroenterol. 108: 1082-1093.

71. El-Serag, H.B., J.M. Hollier, P. Gravitt, et al. 2013. Human papillomavirus and the risk of Barrett's esophagus. Dis. Esophagus. 26: 517-521.

72. Rai, N., G.J. Jenkins, E. McAdam, et al. 2008. Human papillomavirus infection in Barrett's oesophagus in the UK: an infrequent event. J. Clin. Virol. 43: 250-252.

73. Iyer, A., V. Rajendran, C.S. Adamson, et al. 2011. Human papillomavirus is detectable in Barrett's esophagus and esophageal carcinoma but is unlikely to be of any etiologic significance. J. Clin. Virol. 50: 205-208.

74. Antonsson, A., L. Knight, D.C. Whiteman, et al. 2016. Human papillomavirus not detected in esophageal adenocarcinoma tumor specimens. Cancer Epidemiol. 41: 96-98.

75. Rajendra, S. \& B. Wang. 2016. Human papillomavirus not detected in esophageal adenocarcinoma tumor specimensletter. Cancer Epidemiol. 43: 119.

76. Acevedo-Nuno, E., A. Gonzalez-Ojeda, G. VazquezCamacho, et al. 2004. Human papillomavirus DNA and protein in tissue samples of oesophageal cancer, Barrett's oesophagus and oesophagitis. Anticancer Res. 24: 13191323.

77. Li, X., C. Gao, Y. Yang, et al. 2014. Systematic review with meta-analysis: the association between human papillomavirus infection and oesophageal cancer. Aliment Pharmacol. Ther. 39: 270-281.

78. Kunzmann, A.T., S. Graham, C.M. McShane, et al. 2017. The prevalence of viral agents in esophageal adenocarcinoma and Barrett's esophagus: a systematic review. Eur. J. Gastroenterol. Hepatol. 29: 817-825.

79. Wang, B., S. Rajendra, D. Pavey, et al. 2013. Viral load and integration status of high-risk human papillomaviruses in the Barrett's metaplasia-dysplasia-adenocarcinoma sequence. Am. J. Gastroenterol. 108: 1814-1816.

80. Rajendra, S., B. Wang, D. Pavey, et al. 2015. Persistence of human papillomavirus, overexpression of p53, and outcomes of patients after endoscopic ablation of Barrett's esophagus. Clin. Gastroenterol. Hepatol. 13: 1364-1368. e1365.

81. Braakhuis, B.J., P.J. Snijders, W.J. Keune, et al. 2004. Genetic patterns in head and neck cancers that contain or lack transcriptionally active human papillomavirus. J. Natl. Cancer Inst. 96: 998-1006.

82. Rajendra, S., B. Wang, N. Merrett, et al. 2016. Genomic analysis of HPV-positive versus HPV-negative oesophageal adenocarcinoma identifies a differential mutational landscape. J. Med. Genet. 53: 227-231.

83. Rajendra, S., T. Yang, W. Xuan, et al. 2017. Active human papillomavirus involvement in Barrett's dysplasia and oesophageal adenocarcinoma is characterized by wild-type 
p53 and aberrations of the retinoblastoma protein pathway. Int. J. Cancer 141: 2037-2049.

84. Spechler, S.J. \& R.F. Souza. 2014. Barrett's esophagus. N. Engl. J. Med. 371: 836-845.

85. Stanfield, B.A. \& M.A. Luftig. 2017. Recent advances in understanding Epstein-Barr virus. F1000Res. 6: 386.

86. Young, L.S. \& A.B. Rickinson. 2004. Epstein-Barr virus: 40 years on. Nat. Rev. Cancer 4: 757-768.

87. Tsao, S.W., C.M. Tsang, K.F. To, et al. 2015. The role of Epstein-Barr virus in epithelial malignancies. J. Pathol. 235: 323-333.

88. Vockerodt, M., L.F. Yap, C. Shannon-Lowe, et al. 2015. The Epstein-Barr virus and the pathogenesis of lymphoma. J. Pathol. 235: 312-322.

89. Lassmann, H., G. Niedobitek, F. Aloisi, et al. 2011. EpsteinBarr virus in the multiple sclerosis brain: a controversial issue-report on a focused workshop held in the Centre for Brain Research of the Medical University of Vienna, Austria. Brain 134: 2772-2786.

90. Chang, Y., C.-H. Tung, Y.-T. Huang, et al. 1999. Requirement for cell-to-cell contact in Epstein-Barr virus infection of nasopharyngeal carcinoma cells and keratinocytes. J. Virol. 73: 8857-8866.

91. Gulley, M.L. 2015. Genomic assays for Epstein-Barr viruspositive gastric adenocarcinoma. Exp. Mol. Med. 47: e134.

92. Shinozaki-Ushiku, A., A. Kunita \& M. Fukayama. 2015. Update on Epstein-Barr virus and gastric cancer (review). Int. J. Oncol. 46: 1421-1434.

93. Iizasa, H., A. Nanbo, J. Nishikawa, et al. 2012. EpsteinBarr Virus (EBV)-associated gastric carcinoma. Viruses 4: 3420-3439.

94. Cancer Genome Atlas Research Network. 2014. Comprehensive molecular characterization of gastric adenocarcinoma. Nature 513: 202-209.

95. Cancer Genome Atlas Research Network, Analysis Working Group: Asan University, BC Cancer Agency, et al. 2017. Integrated genomic characterization of oesophageal carcinoma. Nature 541: 169-175.

96. Genitsch, V., A. Novotny, C.A. Seiler, et al. 2015. EpsteinBarr virus in gastro-esophageal adenocarcinomas-single center experiences in the context of current literature. Front Oncol. 5: 73.

97. Wu, M.Y., X.Y. Wu \& C.X. Zhuang. 2005. Detection of HSV and EBV in esophageal carcinomas from a high-incidence area in Shantou China. Dis. Esophagus. 18: 46-50.

98. Awerkiew, S., A.Z. Hausen, S.E. Baldus, et al. 2005. Presence of Epstein-Barr virus in esophageal cancer is restricted to tumor infiltrating lymphocytes. Med. Microbiol. Immunol. 194: 187-191.

99. Chen, P.C., C.C. Pan, W.H. Hsu, et al. 2003. Epstein-Barr virus-associated lymphoepithelioma-like carcinoma of the esophagus. Hum. Pathol. 34: 407-411.

100. Valbuena, J.R., Y. Retamal, C. Bernal, et al. 2007. EpsteinBarr virus-associated primary lymphoepitheliomalike carcinoma of the esophagus. Diagn. Mol. Pathol. 16: 27-31.

101. Terada, T. 2013. Epstein-Barr virus associated lymphoepithelial carcinoma of the esophagus. Int. J. Clin. Exp. Med. 6: 219-226.
102. Polyomaviridae Study Group of the International Committee on Taxonomy of Viruses, S. Calvignac-Spencer, Feltkamp, M.C., et al. 2016. A taxonomy update for the family Polyomaviridae. Arch. Virol. 161: 1739-1750.

103. Iaconelli, M., S. Petricca, S.D. Libera, et al. 2015. First detection of human papillomaviruses and human polyomaviruses in river waters in Italy. Food Environ. Virol. 7: 309-315.

104. Moens, U., K. Rasheed, I. Abdulsalam, et al. 2015. The role of Merkel cell polyomavirus and other human polyomaviruses in emerging hallmarks of cancer. Viruses 7: 1871-1901.

105. Chang, Y. \& P.S. Moore. 2012. Merkel cell carcinoma: a virus-induced human cancer. Annu. Rev. Pathol. 7: 123144.

106. Loyo, M., R. Guerrero-Preston, M. Brait, et al. 2010. Quantitative detection of Merkel cell virus in human tissues and possible mode of transmission. Int. J. Cancer 126: 29912996.

107. Yahyapour, Y., F. Sadeghi, A. Alizadeh, et al. 2016. Detection of Merkel cell polyomavirus and human papillomavirus in esophageal squamous cell carcinomas and non-cancerous esophageal samples in Northern Iran. Pathol. Oncol. Res. 22: 667-672.

108. Padgett, B.L., D.L. Walker, G.M. ZuRhein, et al. 1971. Cultivation of papova-like virus from human brain with progressive multifocal leucoencephalopathy. Lancet 1: $1257-1260$.

109. Ricciardiello, L., D.K. Chang, L. Laghi, et al. 2001. Mad1 is the exclusive JC virus strain present in the human colon, and its transcriptional control region has a deleted 98-base-pair sequence in colon cancer tissues. J. Virol. 75: 1996-2001.

110. Enam, S., L. Del Valle, C. Lara, et al. 2002. Association of human polyomavirus JCV with colon cancer: evidence for interaction of viral T-antigen and beta-catenin. Cancer Res. 62: 7093-7101.

111. Del Valle, L., M.K. White, S. Enam, et al. 2005. Detection of JC virus DNA sequences and expression of viral T antigen and agnoprotein in esophageal carcinoma. Cancer 103: 516-527.

112. Del Valle, L., J. Gordon, M. Assimakopoulou, et al. 2001. Detection of JC virus DNA sequences and expression of the viral regulatory protein $\mathrm{T}$-antigen in tumors of the central nervous system. Cancer Res. 61: 4287-4293.

113. Stevens, H.P., D.P. Kelsell, S.P. Bryant, et al. 1996. Linkage of an American pedigree with palmoplantar keratoderma and malignancy (palmoplantar ectodermal dysplasia type III) to $17 \mathrm{q} 24$. Literature survey and proposed updated classification of the keratodermas. Arch. Dermatol. 132: 640651.

114. Kelsell, D.P., J.M. Risk, I.M. Leigh, et al. 1996. Close mapping of the focal non-epidermolytic palmoplantar keratoderma (PPK) locus associated with oesophageal cancer (TOC). Hum. Mol. Genet. 5: 857-860.

115. Howel-Evans, W., R.B. Mcconnell, C.A. Clarke \& P.M. Sheppard. 1958. Carcinoma of the oesophagus with keratosis palmaris et plantaris (tylosis): a study of two families. Q. J. Med. 27: 413-429. 
116. Ellis, A., J.K. Field, E.A. Field, et al. 1994. Tylosis associated with carcinoma of the oesophagus and oral leukoplakia in a large Liverpool family-a review of six generations. Eur. J. Cancer B Oral Oncol. 30B: 102-112.

117. Ellis, A., J.M. Risk, T. Maruthappu, et al. 2015. Tylosis with oesophageal cancer: diagnosis, management and molecular mechanisms. Orphanet J. Rare Dis. 10: 126.

118. Blaydon, D.C., S.L. Etheridge, J.M. Risk, et al. 2012. RHBDF2 mutations are associated with tylosis, a familial esophageal cancer syndrome. Am. J. Hum. Genet. 90: 340-346.

119. Brooke, M.A., S.L. Etheridge, N. Kaplan, et al. 2014. iRHOM2-dependent regulation of ADAM17 in cutaneous disease and epidermal barrier function. Hum. Mol. Genet. 23: 4064-4076.

120. Maney, S.K., D.R. McIlwain, R. Polz, et al. 2015. Deletions in the cytoplasmic domain of iRhom 1 and iRhom 2 promote shedding of the TNF receptor by the protease ADAM17. Sci. Signal 8: ra109.

121. Yamazaki, T., Y. Iwaya, M. Iwaya, et al. 2016. A case of simultaneous esophageal squamous cell carcinoma and Barrett's adenocarcinoma. Clin. J. Gastroenterol. 9: 222-227.

122. Geramizadeh, B., A. Safari, F. Javadi, et al. 2011. Squamous cell carcinoma of the esophagus and adenocarcinoma of gastroesophageal junction, a rare coincidence. Iran Red. Crescent Med. J. 13: 507-509.

123. Rubio, C.A. \& B. Aberg. 1991. Barrett's mucosa in conjunction with squamous carcinoma of the esophagus. Cancer 68: $583-586$

124. Domper Arnal, M.J., A. Ferrandez Arenas \& A. Lanas Arbeloa. 2015. Esophageal cancer: risk factors, screening and endoscopic treatment in Western and Eastern countries. World J. Gastroenterol. 21: 7933-7943.

125. de Jonge, P.J., M. van Blankenstein, W.M. Grady, et al. 2014. Barrett's oesophagus: epidemiology, cancer risk and implications for management. Gut 63: 191-202.
126. Hardikar, S., L. Onstad, P.L. Blount, et al. 2013. The role of tobacco, alcohol, and obesity in neoplastic progression to esophageal adenocarcinoma: a prospective study of Barrett's esophagus. PLoS One 8: e52192.

127. International Agency for Research on Cancer (IARC). 2000. World Health Organization Classification of Tumours. Lyon, France: IARCPress.

128. Mishima, Y., Y. Amano, T. Yuki, et al. 2011. A rare case of Barrett's adenocarcinoma including squamous cell carcinoma component. Clin. J. Gastroenterol. 4: 5-9.

129. van Rees, B.P., R.W. Rouse, M.J. de Wit, et al. 2002. Molecular evidence for the same clonal origin of both components of an adenosquamous Barrett carcinoma. Gastroenterology 122: 784-788.

130. Sharma, P., T.G. Morales, A. Bhattacharyya, et al. 1998. Squamous islands in Barrett's esophagus: what lies underneath? Am. J. Gastroenterol. 93: 332-335.

131. Gore, S., C.J. Healey, R. Sutton, et al. 1993. Regression of columnar lined (Barrett's) oesophagus with continuous omeprazole therapy. Aliment Pharmacol. Ther. 7: 623628 .

132. Malesci, A., V. Savarino, P. Zentilin, et al. 1996. Partial regression of Barrett's esophagus by long-term therapy with high-dose omeprazole. Gastrointest. Endosc. 44: 700705 .

133. Garewal, H., L. Ramsey, P. Sharma, et al. 1999. Biomarker studies in reversed Barrett's esophagus. Am. J. Gastroenterol. 94: 2829-2833.

134. Allende, D., J. Dumot \& L. Yerian. 2013. Esophageal squamous cell carcinoma arising after endoscopic ablation therapy of Barrett's esophagus with high-grade dysplasia. Report of a case. Dis. Esophagus 26: 314-318.

135. Zeki, S.S., R. Haidry, M. Justo-Rodriguez, et al. 2014. Squamous cell carcinoma after radiofrequency ablation for Barrett's dysplasia. World J. Gastroenterol. 20: 44534456. 